\begin{tabular}{|c|c|}
\hline Title & Mechanisms of photoinduced fluidity in chal cogenide glasses: Molecular orbital analy ses \\
\hline Author(s) & Tanaka, Keiji; Shimakawa, Koichi \\
\hline Citation & $\begin{array}{l}\text { Journal of Non-Crystalline Solids, 481, 579-584 } \\
\text { https://doi.org/10.1016/.jnoncrysol.2017.12.005 }\end{array}$ \\
\hline Issue Date & 2018-02-01 \\
\hline Doc URL & http:/hdl.handle.net/2115/76642 \\
\hline Rights & $\begin{array}{l}\text { (0) 2018. This manuscript version is made available under the CC-BY-NC-ND } 4.0 \text { license } \\
\text { http://creativecommons.org/icenses/by-nc-nd/4.0/ }\end{array}$ \\
\hline Rights(URL) & http://creativecommons.org/icenses/by-nc-nd/4.0/ \\
\hline Type & article (author version) \\
\hline File Information & Tanaka Shimakawa.pdf \\
\hline
\end{tabular}

Instructions for use 


\title{
Mechanisms of photoinduced fluidity in chalcogenide glasses: molecular orbital analyses
}

Keiji Tanaka*,1 and Koichi Shimakawa ${ }^{2}$

${ }^{1}$ Department of Applied Physics, Graduate School of Engineering, Hokkaido University, Sapporo 060-8628, Japan

${ }^{2}$ Department of Electrical and Electronic Engineering, Gifu University, Gifu 501-1193, Japan

\begin{abstract}
Atomic processes giving rise to the photoinduced fluidity have been studied for amorphous selenium. $A b$ initio molecular orbital calculations of Se clusters suggest that distorted atomic structures such as curled and intersecting chains have smaller optical gaps. Those atomic sites are likely to be excited by subgap (Urbach-edge) light, which undergo structural relaxation including bond breakages and interchanges. Such photo-electro-structural transformations could originate the macroscopic fluidity. Possibility of electronic glass-transition is also discussed.
\end{abstract}

Classifications: 30: amorphous semiconductors, 190: glasses, 310: optical properties, 480: Viscosity and relaxation

Keywords: Se, photoinduced, viscosity, GAMESS, dangling bond

*Corresponding author: e-mail keij@@eng.hokudai.ac.jp 


\section{Introduction}

Glassy materials are known to behave fluidally under electronic excitations, the fact being reported by Vonwiller for Se about a century ago [1]. Recent studies have demonstrated that such fluidity $f(=1 / \eta$, where $\eta$ is the viscosity) appears also in $\mathrm{SiO}_{2}$ at temperatures substantially below the glass-transition temperature [2]. Besides, $\mathrm{Se}, \mathrm{As}_{2} \mathrm{Se}_{3}, \mathrm{As}_{2} \mathrm{~S}_{3}$ and Ge-Se exhibit photoinduced fluidity* at low temperatures [3-8], which manifests its athermal nature. We may then regard that the phenomenon is a kind of "electronic melting", observed in crystals under intense, pulsed light exposures [9-11]. Nevertheless, it should be emphasized that the fluidity in glasses is conspicuous under moderate excitations. Specifically, in the chalcogenide glasses with bandgap energies of $\sim 2 \mathrm{eV}$ [12], the fluidity of $\sim 10^{-12} \mathrm{P}^{-1}$ appears during illumination of subgap photons, or so-called Urbach-edge light possessing absorption coefficients of $1-10^{4}$ $\mathrm{cm}^{-1}$, emitted from $10 \mathrm{~mW}$-class $\mathrm{cw}$ lasers [4-8]. In applications, this phenomenon has potential for optical micro-fabrication [8, 13]. Also, the fluidity in combination with several kinds of motive forces is able to produce permanent photoinduced deformations [14, 15], which may be promising to make optical devices such as micro lenses [15] and polarization-controlled structures [16]. However, why the glass exhibits the electronically induced fluidity remains speculative.

Footnote *The phenomenon that a chalcogenide glass becomes fluidal during (not after) expositions to light has been termed in several ways, such as photoinduced stress-relaxation [7] and viscosity [6, 14], which are unified into "fluidity" in the present work.

(a)

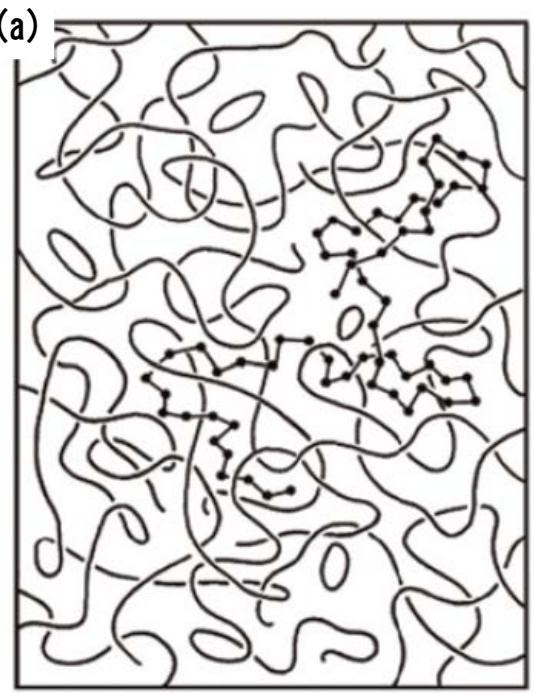

(b)

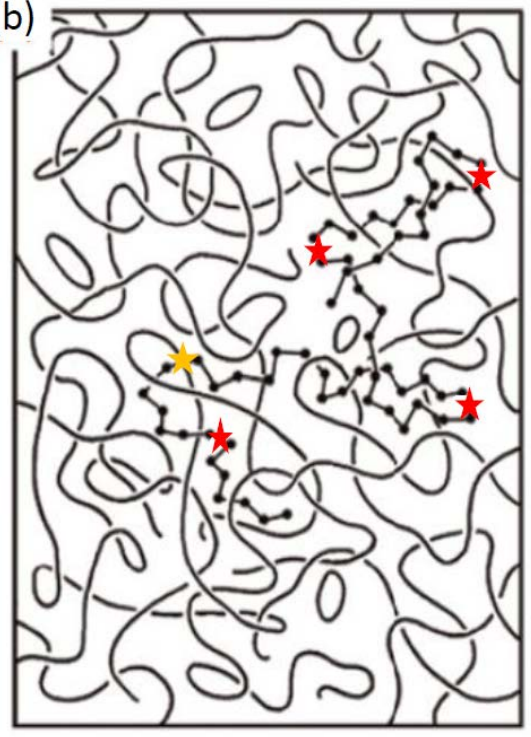

Fig. 1. An entangled chain/ring structure of a-Se [12], in which the atoms are depicted only on a single chain for simplicity. (a) shows an annealed state, and (b) its structure under a vertical stress (exaggerated) and illumination, with the red and orange stars representing excited sites, which may undergo bond breakages and intersections, respectively.
We here focus upon amorphous (a-) Se as the simplest example, with a schematic atomic view being given in Fig. 1(a) as a reference [12]. The structure is composed only with -Se-Se- homopolar bonds, which probably form (deformed) rings and entangled chains, the ratio naturally depending upon preparation procedures [17]. In the meta-stable structures, however, the short-range order, i.e. the coordination number $Z(=2)$, bond length $r(\approx 2.3 \AA)$ and bond angle $\theta\left(\approx 105^{\circ}\right)$, is mostly preserved, with some disorder in 
the dihedral angle $\varphi$ and the inter-chain distance $R[18,19]$. A single chain may be longer than $10^{5}$ in the atom number $[12,18]$. Native defects such as $\mathrm{C}_{3}$ (three-fold coordinated chalcogen) could also exist, though the estimated density might be too few to directly affect mechanical properties [12].

We note here that such a structure as in Fig. 1 resembles those in organic polymers, a typical substance being polyethylene PE, comprising of $-\mathrm{CH}_{2}-$. For its amorphous states, substantial studies on atomic motions have been performed, which develop principal concepts such as the Rouse and the reptation model [20-24]. Hence, those outcomes could give insights into the dynamics of a-Se.

Nevertheless, we should also mark two characteristic differences between the organic and inorganic polymers: One is the bond strengths. For instance, the Pauling's bond energies for C-C and Se-Se are 3.6 and $1.9 \mathrm{eV}$. Besides, the inter- and intra-molecular distance ratios $R / r$ in PE and Se are $\sim 3.3(\approx 5 / 1.5 \AA$ [ 21 , $23])$ and $\sim 1.5(\approx 3.7 / 2.4 \AA[18,19])$. In PE the intra- and the inter-molecular characters are distinctly different, and accordingly, chain crossing upon structural changes cannot be a common process [20, 22, 24], which may not be the case in liquid Se [25] (and also in liquid S [26]). The other regards the electronic structures. In contrast to the sp3-type valence-electron configuration of $\mathrm{C}$ atoms in $\mathrm{PE}$, Se atoms in the $\mathrm{C}_{2}$ (two-fold coordinated) bonding retain the $\mathrm{p}^{4}$-like wavefunction, which splits into the $\sigma, \pi-\pi^{*}$, and $\sigma^{*}$ states. In many Se clusters, these $\pi^{*}$ and $\sigma^{*}$ states form the HOMO and LUMO levels, which in the elemental lone-pair (LP) electron semiconductor [12], solid Se, constitute the edges of the valence and the conduction band with the optical gap of $\sim 2.0 \mathrm{eV}$ [18].

When a-Se as in Fig. 1(a) is illuminated under stresses, what kinds of atomic motions occur? It might be assumed that the stress produces elastically-strained structures, which are altered by illumination. However, such distortions seem to be practically neglected, due to small applied forces; the elastic strains in reported experiments being only at levels of $10^{-3}-10^{-2}$ [3-8]. In addition, thermal disorder effects could also be neglected, since the photoinduced fluidity is conspicuous at low temperatures $(\geq 220 \mathrm{~K})$ [3-8]. These situations may put forward the following scenario; Urbach-edge light excites LP electrons at disordered atomic sites having smaller, local optical gaps, and then the sites athermally undergo structural changes such as chain breakages, which release local strains and cause fluidity under weak external forces. We therefore first examine what kinds of disordered structures can possess smaller optical gaps, and next how the excited sites undergo structural changes. Note that, to the author's knowledge, no computational studies have been performed on electronic excitation effects in PE-like polymers, despite of extensive works on dye-doped polymers [27, 28].

\section{Modeling and Calculations}

Substantial theoretical studies have been performed for electronic and structural properties of disordered chain/ring(-like) a-Se structures. For instance, molecular-dynamics (MD) calculations have provided valuable insights into global electro-structural evolutions [29-32]. Nevertheless, the method is inadequate to analyze elemental processes. Also, several challenging ideas trying to connect atomic structures to macroscopic viscosity have been presented [33-36], while it seems difficult to apply such approaches to electronically excited states. 
Taking these backgrounds into account, we here analyze simple clusters, $\mathrm{H}^{-} n \mathrm{Se}-\mathrm{H}$ with $n$ being an integer, using a molecular-orbital (MO) method. The terminal $\mathrm{H}$ is attached to compensate dangling-bond effects, and it may also represent a connected chain. The local optical gap could be estimated from the exciton-excitation energy $E_{\text {exc }}$ (or the HOMO-LUMO energy $E_{\mathrm{HL}}$ ) in strained Se clusters. Specifically, we examine structure-energy correlations mainly for three clusters: i) $\mathrm{H}-4 \mathrm{Se}-\mathrm{H}$, which is the shortest chain possessing necessary structural factors $(r, \theta$ and $\varphi)$ characterizing longer chains [37, 38], and accordingly, it could typify a chain segment in a-Se. ii) A dimer consisting of $\mathrm{H}-\mathrm{Se}-\mathrm{H}$ and $\mathrm{H}-3 \mathrm{Se}-\mathrm{H}$, which also contains four Se atoms for comparison. Disorder is likely to exist in varied inter-cluster atomic distances $R$. And, iii) a pair of $\mathrm{H}-2 \mathrm{Se}-\mathrm{H}$, in which the inter-cluster distance may be reduced by viscous motions, and the pair ultimately overcomes intercrossing. Such a process is inevitable to release knots in entangled chains.

Employed softwares were an ab initio molecular orbital package GAMESS [39] operating on a graphic platform Winmoster [40], the details being described previously [37, 38]. In short, a selected base function was of the $6-31+\mathrm{G}^{*}$ type and the B3LYP-DFT approximation was adopted, the combination having been demonstrated to provide satisfactory results with acceptable computation times ( $\leq 1$ day). Equilibrium shapes were obtained through the energy-optimization calculation of the total energy $E_{\Gamma}$, and the excitonic state with $E_{\text {exc }}$ was analyzed using the time-dependent DFT routine in GAMESS.

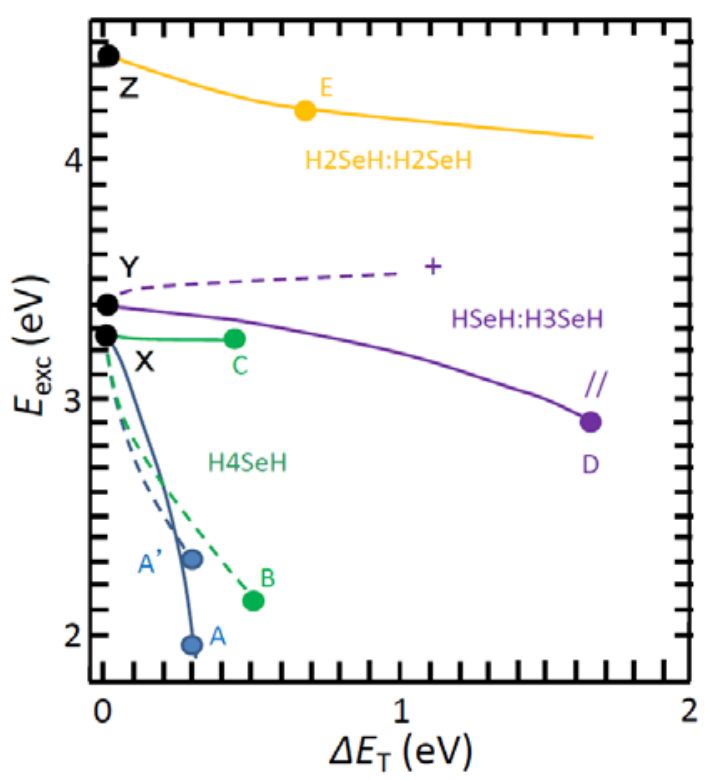

Fig. 2. The excitonic energy $E_{\text {exc }}$ as a function of the increase $\Delta E_{\Gamma}$ (strain) in the total energy for the three clusters; H4SeH (lower), $\mathrm{HSeH}: \mathrm{H} 3 \mathrm{SeH}$ (middle), and paired H2SeH (upper). For the points X, A, A', etc., see the text.

\section{Results}

Fig. 2 shows $E_{\text {exc }}$ as a function of the strain energy $\Delta E_{\mathrm{T}}$ for the three clusters. At the equilibrium (X in Fig. 2, Fig. 3(a)), H-4Se-H has a helical structure with $r=$ $2.37 \AA, \theta=107^{\circ}$ and $\varphi=87^{\circ}$, which give the distance $L$ between the far-side Se atoms at $4.9 \AA$ and $E_{\text {exc }}=3.27$ $\mathrm{eV}\left(E_{\mathrm{HL}}=4.03 \mathrm{eV}\right)$, in consistent with previous results [37, 38]. In the clusters of $\mathrm{H}-\mathrm{Se}-\mathrm{H}: \mathrm{H}-3 \mathrm{Se}-\mathrm{H}$ (Y in Fig. 2, Fig. 4(a)) and paired H-2Se-H (Z in Fig. 2, Fig. 6(a)), the inter-cluster distances have initially been set greater than $\sim 7 \AA$. These three systems possess $E_{\mathrm{T}}$ of -9605.886 , -9607.053 , and $-9600.497 \mathrm{H}$, respectively, which are taken as references at $\Delta E_{\Gamma}=0$ in Fig. 2. The slightly different $E_{\mathrm{T}}$ values, despite of the same number $(=4)$ of Se atoms, reflect structural differences including the cluster sizes and two/four $\mathrm{H}$ atoms. Also, $E_{\text {exc }}$ at the equilibriums are different with the same reason, so that the absolute values of $E_{\text {exc }}$ have little meanings when considering practical situations in a-Se. Note that the figure plots only $E_{\text {exc }}$ for simplicity, since the variations of $E_{\mathrm{exc}}$ and $E_{\mathrm{HL}}$ are roughly parallel, with the latter being greater by $\sim 1 \mathrm{eV}$. 

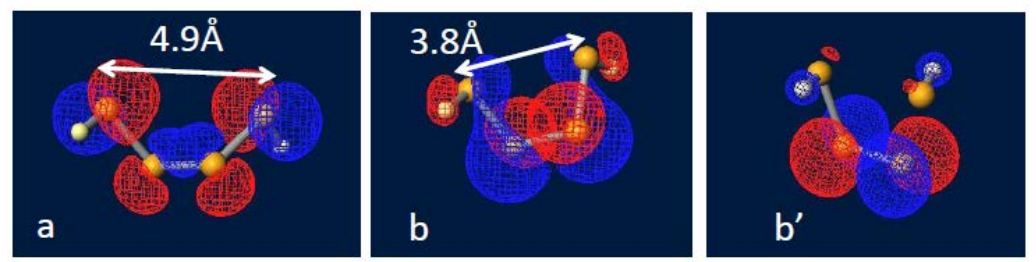

\subsection{Disordered single chain}

Single chains could easily be deformed with bents. The bent, which includes variations of $\varphi$ (and $L$ ), ultimately changes the stable
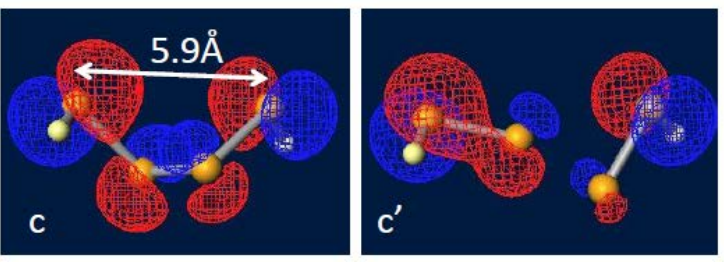
trans-structure with $\varphi \approx 90^{\circ}$ (Fig. 3(a)) to a cis-structure $\left(\varphi=0^{\circ}\right)$ or to a planar zig-zag shape $\left(\varphi=180^{\circ}\right)$.

Fig. 3(b, b') shows a sequential

Fig. 3. Sequences of structural changes of $\mathrm{H}-4 \mathrm{Se}-\mathrm{H}$, with HOMO wavefunctions attached. (a) shows the optimized helical conformation, (b) a cis-structure, which is excited and energy-optimized in (b'), and (c) a stretched structure, which is excited and energy-optimized in (c').

response of $\mathrm{H}-4 \mathrm{Se}-\mathrm{H}$ having the cis-conformation. In (b), the structure has been energy-optimized under a constraint of $L=3.8 \AA$. In such structures, as illustrated, the LP wavefunctions of the central two Se atoms align in anti-parallel, which enhances the LP-LP interaction, increases the HOMO energy, and gives rise to a dramatic reduction of $E_{\text {exc }}$, as plotted at $\mathrm{A}$ in Fig. 2 . The increase in $\varphi$ to a planar zig-zag shape has also undergone a similar electronic change, to the state A'. Such atomic sites having small $E_{\text {exc }}$ are likely to be excited by subgap photons, the process having been analyzed through the energy-optimized time-dependent DFT calculation. The result demonstrates a conformational transformation from Fig. 3(b) to (b'), i.e., scission ( $r \geq 2.7 \AA)$ of a bond. After recombination of the exciton, however, the dangling bonds tend to recombine. Here, it should be noted that the small $\Delta E_{\mathrm{T}}$ values of A and A' imply the existence of a lot of bent and planar chains in a-Se.

Fig. 3 also includes a sequential response of a stretched H-4Se-H. In Fig. 3(c), $L$ has been elongated to $5.9 \AA$ and energy-optimized, the result giving rise to a structure; $r=2.39-2.65 \AA, \theta=114^{\circ}$ and $\varphi=107^{\circ}$ with $\Delta E_{\mathrm{T}}=0.5 \mathrm{eV}$ and $E_{\mathrm{exc}}=2.14 \mathrm{eV}, \mathrm{B}$ in Fig. 2 . The $E_{\mathrm{exc}}$ reduction by $\sim 1 \mathrm{eV}$ from the equilibrium shape is governed by rising HOMO level, again arising from enhanced LP-LP interaction. Then, this stretched structure has been excited under the fixed $L$ and energy-optimized, the result being shown in Fig. 3(c'), which manifests scission $(r \approx 2.8 \AA$ ) of the central bond. Qualitatively the same change has been obtained for H-5Se-H. Note that the bond disruption has occurred also in $L$-constrained planar zig-zag chains (not shown), but never occurred in unconstraint helical chains (Fig. 3(a)). These observations imply that, in entangled a-Se structures, distorted segments are susceptible to bond breakage, which can release accumulated strains.

In passing, it should be noted that axially compressed $\mathrm{H}-4 \mathrm{Se}-\mathrm{H}$ exhibits little $E_{\text {exc }}$ changes. For instance, the structural parameters at $\mathrm{C}$ in Fig. 2 are $L=3.92 \AA, r=2.35 \sim 2.40 \AA, \theta=101^{\circ}$, and $\varphi=54^{\circ}$ with $\Delta E_{\mathrm{T}}=0.43 \mathrm{eV}$ and $E_{\mathrm{exc}}=3.43 \mathrm{eV}$. (Further $L$ reduction has resulted in chain bending, which may be a signature of buckling.) It should also be emphasized that no bond breaking has occurred in the compressed $\mathrm{H}-4 \mathrm{Se}-\mathrm{H}$. Probably, the bond elongation, which could be induced by the excitation, is 
suppressed by the constraint on $L$.

\subsection{Double chains with varied inter-cluster distances}

Fig. 2 also shows $E_{\text {exc }}$ variations in the dimer H-Se-H:H-3Se-H. $R$ is reduced from $\sim 7$ (Y in Fig. 2) to $2.5 \AA$, the range covering the (estimated) interchain distance of $\sim 3.7 \AA$ [19] in a-Se. For the dimer, we presume two types of inter-cluster LP-LP conformations; parallel (//) (Fig. 4(a)) and orthogonal (+) (Fig. $5(\mathrm{a})$ ), which could be fixed in the present calculation by the positions of the two $\mathrm{H}$ atoms of the $\mathrm{H}-\mathrm{Se}-\mathrm{H}$ cluster.

We see in Fig. 2 that the parallel LP dimer undergoes a substantial $E_{\text {exc }}$ decrease with the $R$ reduction. And at $R \approx 2.5 \AA$, the point D in Fig. 2, the cluster produces two neutral $\mathrm{C}_{3}$ (Fig. 4(b)), a cross-linker. (Here, a big $\Delta E_{\Gamma}$ at the point should not be taken seriously, which may become smaller in longer chains having more interchain-interacting sites.) The LP-LP interaction becomes enhanced, which rises the HOMO level by $\sim 0.4 \mathrm{eV}$ with minor lowering $(\sim 0.15 \mathrm{eV})$ of the LUMO level, the result giving rise to a marked $E_{\text {exc }}$ reduction from 3.4 to $2.9 \mathrm{eV}$. Note that this correlation between $R$ and $E_{\text {exc }}$ is consistent with results of pressure experiments, i.e. volume compaction and red-shifting optical absorption edge in g-Se under
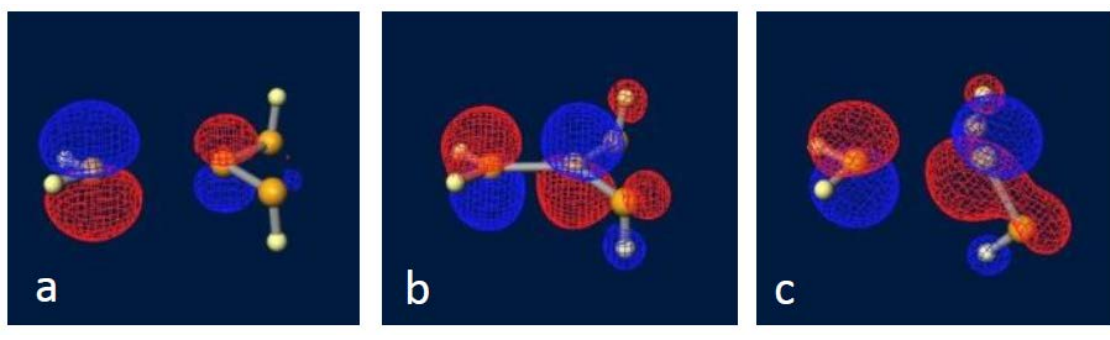

Fig. 4 The HOMO wavefunction of the dimer consisting of $\mathrm{H}-\mathrm{Se}-\mathrm{H}: \mathrm{H}-3 \mathrm{Se}-\mathrm{H}$ with the parallel LP interaction at (a) $R=4.0 \AA$, (b) $2.5 \AA$, and (c) its excited state.
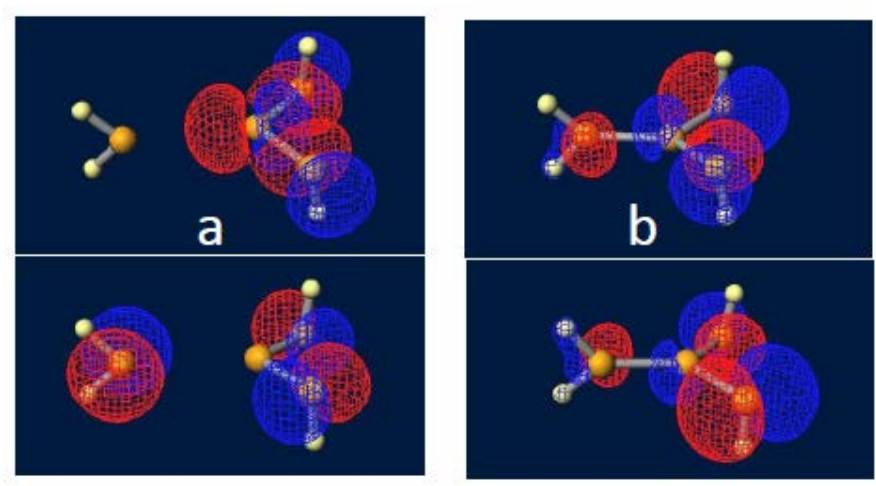

Fig. 5. HOMO (lower) and LUMO (upper) wavefunctions of $\mathrm{H}-\mathrm{Se}-\mathrm{H}: \mathrm{H}-3 \mathrm{Se}-\mathrm{H}$ with the orthogonal LP interaction at (a) $R=4.0 \AA$ and (b) $2.5 \AA$. hydrostatic compressions [12, 41].

Light excitation is likely to take place at such small $E_{\text {exc }}$ sites. The induced process has been analyzed under fixing the positions of the four terminal $\mathrm{H}$ atoms. The resulting structure, shown in Fig. 4(c), contains two $\mathrm{C}_{1}$ atoms which are separated by $\sim 4 \AA$. The dimer has been fragmented, which implies that cross-linked chains in a-Se may also undergo such fragmentation.

On the other hand, as shown in Fig. 5, the orthogonal LP sites exhibit a contrastive behavior. Upon reducing $R$, as in Fig. 5(b), $\mathrm{C}_{3}$ pairs are forced to be produced with a small rise in the HOMO energy from -6.7 to $-6.4 \mathrm{eV}$, which suggests enhanced LP-LP interaction. Concomitantly, however, the LUMO level also rises from -2.4 to $-2.1 \mathrm{eV}$, reflecting repulsive interaction. In consequence, as shown in Fig. $2, E_{\text {exc }}$ hardly 
changes (or rather increases) under the $R$ reduction. Accordingly, such sites are unlikely to absorb subgap light. Probably, the orthogonal LP sites are less responsible for photoinduced transformations in a-Se.

\subsection{Chain Crossing}

Fluidal motions of segmental chains may ultimately be hindered by chain entanglements. To analyze such situations, we first produce two $\mathrm{H}-2 \mathrm{Se}-\mathrm{H}$ clusters, which are orthogonally aligned each other, along the $x$ and $y$ directions, and are separated sufficiently $(R \sim 7 \AA)$ in the $z$ direction. The structure has been energy-optimized, the result giving the total energy of $-4800.249 \times 2 \mathrm{H}$, which is taken at $\Delta E_{\mathrm{T}}=0 \mathrm{eV}, \mathrm{Z}$ in Fig. 2 . Then, the separation is gradually reduced, which increases $E_{\mathrm{T}}$ and reduces $E_{\text {exc }}$, both reflecting the inter-cluster LP-LP interaction. For instance, Fig. 6(a) shows the structure at separation of $\sim 3.0 \AA$ and $\Delta E_{\mathrm{T}}=0.7 \mathrm{eV}$, corresponding to $\mathrm{E}$ in Fig. 2. If the separation is further reduced to $\sim 2 \AA, \Delta E_{\mathrm{T}}$ increases to $\sim 8$ $\mathrm{eV}$, much greater than typical thermal energy; e.g., $\sim 30 \mathrm{meV}$ at the glass-transition temperature of $\sim 35^{\circ} \mathrm{C}$ [18]. Such high strain energies practically suppress the chain inter-crossing, in similar ways to those in PE [20]. We may then envisage that some electro-structural excitation assists the crossing.

Calculations for several chain-crossing processes have suggested that the sequence depicted in Fig. 6, the idea proposed by Koseki and Odajima [3], seems to be the most plausible. For instance, the structure shown in Fig. 6(a) may be optically excited. Then, in Fig. 6(b), the excited-state relaxation has produced a $\mathrm{C}_{3}{ }^{+}$and $\mathrm{C}_{1}{ }^{-}$pair (the Mulliken's atomic charges of +0.3 and -0.8 ) with separation of $\sim 3.0 \AA$ A. The excited structure would be temporally held, during which the arrowed atom in Fig. 6(c) could make a thermal twisting motion to a bistable position. Such a motion, which may depend upon the H positions, can occur due to lowered energy barriers between (b) and (c) structures to a few $10 \mathrm{meV}$, reflecting hybridization of relevant wavefunctions and peripheral chain conformations. Note that this energy is comparable to the activation energy of $\sim 0.1 \mathrm{eV}$ experimentally observed in a-Se/mica samples at $\sim 250 \mathrm{~K}$ [3]. (This thermal activation idea may imply that the photoinduced fluidity in a-Se disappears at cryogenic temperatures, which remains to be studied.) Otherwise, the motion may be assisted by peripheral chain movements under external forces, which is difficult to analyze in the present routine. After the twisting motion, the excitation terminates, and (c) relaxes to (d). In short, a photon excitation can assist chain crossing through the bond interchange via temporal production of a valence-alternation pair.
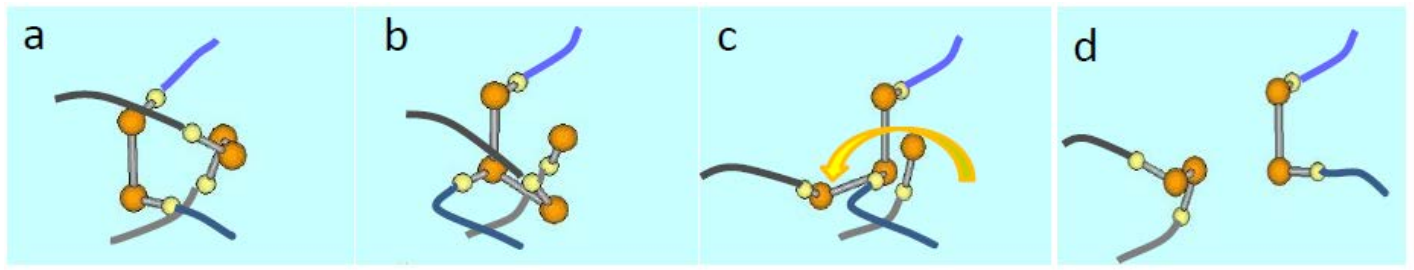

Fig. 6. A chain crossing process: (a) two orthogonal chains are approaching to $\sim 3.0 \AA$ in separation, (b) an optical excitation produces an exciton, which relaxes to a $\mathrm{C}_{3}{ }^{+}$and $\mathrm{C}_{1}{ }^{-}$pair, (c) the atom makes a thermal flip, and (d) the exciton recombines. Lines represent peripheral chain connections. 


\section{Discussion}

Fig. 2 suggests that, among several distorted structures, the bent (A) and the stretched (A' and B) chains are most likely to be photo-fragmented, with the two necessary conditions: One is its population, which is estimated from $N_{0} \exp \left(-\Delta E_{\mathrm{\Gamma}} / k_{\mathrm{B}} T_{\mathrm{g}}\right)$, where $N_{0}$ is the atomic density $\sim 3 \times 10^{22} \mathrm{~cm}^{-3}$ in a-Se and $T_{\mathrm{g}}$ is the glass-transition temperature, $k_{\mathrm{B}} T_{\mathrm{g}} \approx 30 \mathrm{meV}$ [18]. The results in Fig. 2 represent $\Delta E_{\mathrm{T}} \approx 0.2 \mathrm{eV}$ as a typical strain level of the distorted structures, which gives the density of excitable atomic sites at $\sim 10^{19}$ $\mathrm{cm}^{-3}$. Note that this density is comparable with that of the so-called photoinduced ESR; e.g., metastable electron spin signals with densities of $\sim 10^{20} \mathrm{~cm}^{-3}$ in a-Se under illumination at $77 \mathrm{~K}$ [42]. The other is that such sites possess small $E_{\text {exc }}$, which implies that those can be excited by Urbach-edge light with $\alpha^{-1} \geq 1 \mu \mathrm{m}$. We then envisage that the starred bonds in Fig. 1(b) are photo-excited, ruptured, and resulting shorter chains cause the photoinduced fluidity. The model is also in line with the photo-crystallization, a phenomenon more-or-less unique to the elemental a-Se [12], in which the fragmentation could prelude crystalline nucleation. Incidentally, the consideration here may predict that photoinduced effects appear also in strained, single-crystalline trigonal Se. We may also assume that the distorted sites in a-Se behave as dyes in azo-doped polymers in a sense that both species trigger photoinduced mechanical changes, while no bond breakages take place in the azo-polymers [27, 28].

In addition, Fig. 2 manifests roles of the inter-chain interaction. Strained inter-chain sites with parallel LP wavefunctions, exemplified by the point D, are fragmented by light excitation (Fig. 4). By contrast, the site having orthogonal LP wavefunctions is little effective to the photoinduced fluidity (Fig. 5). The chain inter-crossing (Fig. 6), which has been proposed previously [3, 12], seems to play a decisive role under ultimate situations. It is tempting to speculate that the bond breakage governs microscopic fluidity, which is affected by the chain crossing in wider scales.

It should be noted however that the present result, demonstrating the photoinduced chain breakage, may be inconsistent with two previous results. One is an MD result that demonstrates bond scission in electronically-excited unconstrained single Se chains [29], which has not occurred in the present MO calculations. The difference is ascribable to the temperatures; fixed at $500 \mathrm{~K}$ in the MD study [29] and formally $0 \mathrm{~K}$ in MO analyses. Since the photoinduced fluidity appears at low temperatures [2-8], the MO result seems to be more pertinent. (In a unified view, the MD [29] and the present MO result imply that the chain breakage is likely to occur at thermally- and structurally-disordered sites.) The other is that the present result is seemingly in conflict with an EXAFS observation at $30 \mathrm{~K}$ [43]; the fragmentations in Figs. 3 and 4 suggest reductions of the coordination number of $\sim 2$ during illumination, which contrasts with the experimental result demonstrating an increase in the atomic coordination number by $\sim 4 \%$. Reasons of this opposite changes are puzzling. We may speculate that the broad first peak of the pair-distribution function in Ref. 43 has caused an overestimation of the coordination number. Otherwise, anisotropic effects [44] may be responsible for the increase.

In the present context, the next subject is to connect the bond breakage to the fluidity $f(=1 / \eta)$. Nevertheless, quantitative analyses are formidable, due to lacks of structural and statistical data. We then follow an insight obtained for organic polymers and liquid $\mathrm{S}$; the so-called Rouse model for 
one-dimensional polymers that predicts $\eta \sim n[21,23,26]$, where $n$ is the average atom number of single chains. The bond scission in Figs. 3 and 4 suggests linear reduction of $n$ with the light intensity $I$, and accordingly, we obtain $f \sim I$, which has been uncovered in previous experiments [3-8], summarized in Table 1, except the highest-intensity point in Ref. 6.

Quantitatively, what situation can we envisage? We first assume that, under an assumption of unity quantum-efficiency of bond breaking, a steady-state density of ruptured bonds $\alpha I \tau$ ( $\alpha$ the absorption coefficient and $\tau$ the lifetime of broken bonds) should be fewer than $\sim 10^{19} \mathrm{~cm}^{-3}$, the value estimated above from $\Delta E_{\mathrm{T}} \approx 0.2 \mathrm{eV}$. Second, tentatively putting $\tau \approx 10^{-3} \mathrm{~s}$, a long-lived lifetime of photoluminescence in a-Se [45], we obtain $\alpha I \leq 10^{22}$ photons $/ \mathrm{cm}^{3} \mathrm{~s}$, which is satisfied in reported experimental results in Table 1 . Third, we adopt $n \approx 10^{5}$ atoms [12] in un-excited a-Se, which gives $10^{17}$ chains $/ \mathrm{cm}^{3}$, since the atomic density is $\sim 10^{22} / \mathrm{cm}^{3}$. These values deduce that light excitation of $\alpha I \tau \approx 10^{19} \mathrm{~cm}^{-3}\left(\sim 10^{2}\right.$ scissions/chain $)$ is able to reduce $n$ to $\sim 10^{3}$. Provided that this chain length affords the viscosity of $\sim 10^{12} \mathrm{P}$ (Table 1), the $\eta \sim n$ proportionality suggests the dark viscosity to be $\sim 10^{14} \mathrm{P}$, which seems to be a plausible value.

Table 1. Experimental results of the photoinduced fluidity in the glasses (listed in historical order). Since the absorbed photon density is difficult to estimate accurately, it is written as an order of magnitude. The stress relaxation time $\tau\left(\approx 10^{3} \mathrm{~s}\right)$ in Ref. 3 has been converted to the viscosity $\eta$ through the Maxwell's relation $\eta=\tau \mu$, where $\mu\left(\approx 4 \times 10^{9} \mathrm{~N} / \mathrm{m}^{2}\right.$ [18]) is the shear modulus.

\begin{tabular}{|l|l|l|l|}
\hline material & $\begin{array}{l}\text { Light intensity I, excitation } \\
\text { photon energy } \hbar \omega \quad \text { (or light } \\
\text { source) }\end{array}$ & $\begin{array}{l}\text { Viscosity, absorbed photon } \\
\text { density }(=\alpha I / \hbar \omega)\end{array}$ & Reference \\
\hline $\mathrm{Se}$ & $\leq 20 \mathrm{~mW} / \mathrm{cm}^{2}, \mathrm{Hg}$ lamp & $\sim 10^{12} \mathrm{P}, 10^{21}$ photons $/ \mathrm{cm}^{3} \mathrm{~s}$ & Koseki and Odajima [3] \\
\hline $\mathrm{As}_{2} \mathrm{~S}_{3}$ & $\leq 10^{2} \mathrm{~W} / \mathrm{cm}^{2}, 2.0 \mathrm{eV}$ & $\sim 10^{12} \mathrm{P}, 10^{22}$ photons $/ \mathrm{cm}^{3} \mathrm{~s}$ & Tanaka [5] \\
\hline $\mathrm{Se}$ & $\leq 20 \mathrm{~mW} / \mathrm{cm}^{2}, 2.0 \mathrm{eV}$ & $\sim 10^{11} \mathrm{P}, 10^{21}$ photons $/ \mathrm{cm}^{3} \mathrm{~s}$ & Repka et al. [6] \\
\hline $\mathrm{GeSe}_{9}$ & $\leq 10 \mathrm{~W} / \mathrm{cm}^{2}, 1.57 \mathrm{eV}$ & $\sim 10^{12} \mathrm{P}, 10^{21}$ photons $/ \mathrm{cm}^{3} \mathrm{~s}$ & Gueguen et al. [7] \\
\hline
\end{tabular}

Can we extend the fragmentation model to compound glasses such as $\mathrm{As}_{2} \mathrm{~S}_{3}$ and Ge-Se? It seems challenging due to the complicated structures including higher-dimensional amorphous networks comprising of hetero- and homo-polar bonds. For instance, Uchino and Elliott have analyzed excited-state structures of $\mathrm{As}_{2} \mathrm{~S}_{3}$ clusters using an ab initio MO software [46], while no strained effects are taken into account. On the other hand, the present result straightforwardly suggests that, in the glasses having network structures, which may be layer-like, an apparent quantum efficiency of the photoinduced fluidity becomes substantially lowered, since breakage of a single bond cannot necessarily cause the fragmentation of networks. Actually, we see in Table 1, that there seems to exist intensity difference of an order of magnitude for inducing the photoinduced fluidity (with a level of $\eta \sim 10^{12} \mathrm{P}$ ) in between a-Se $\left(\sim 10^{21}\right.$ photons $/ \mathrm{cm}^{3} \mathrm{~S}$ [3], which may be overestimated by an order due to the polychromatic spectrum of an ultrahigh pressure $\mathrm{Hg}$ lamp) and the stoichiometric compound $\mathrm{As}_{2} \mathrm{~S}_{3}\left(\sim 10^{22}\right.$ photons $/ \mathrm{cm}^{3} \mathrm{~s}$ [5]). Besides, 
Gueguen et al. have demonstrated that in the Ge-Se system the photoinduced fluidity becomes less efficient with an increase in the coordination number $Z$ from 2.2 to 2.5 [7]. These observations are in harmony with the photoinduced fragmentation model.

Finally, it might be tempting to consider if the photoinduced fluidity is a manifestation of "electronic glass-transition". As known, $\eta(T)$ at $T \geq T_{\mathrm{K}}$, where $T_{\mathrm{K}}(\approx 220 \mathrm{~K}[47])$ is the Kauzmann temperature, can be fitted by the Vogel-Fulcher-Tamman equation, $\eta(T)=\eta_{0} \exp \left\{a /\left(T-T_{\mathrm{K}}\right)\right\}$, where $\eta_{0}$ and $a$ are constants. It is also known that $\eta\left(T_{\mathrm{g}}\right) \approx 10^{13} \mathrm{P}$, where $T_{\mathrm{g}} \approx 300 \mathrm{~K}[18,47]$. As listed in Table 1, photo-excitation can afford $\eta \approx 10^{12} \mathrm{P}$ at room or lower temperatures, which may then suggest photo-electronic lowering of $T_{\mathrm{g}}$ to the temperatures. In addition, taking an empirical relation $\ln T_{\mathrm{g}} \sim Z[12,48,49]$ into account, we notice that the present result demonstrating the photo-fragmentation, i.e. decreases in $Z$, is consistent with the electronic glass-transition hypothesis.

What are the behaviors in other quantities? For the volume, some researchers have demonstrated experimentally [50,51] and computationally [31] that the volume of a-Se expands during light (electronic) excitations. By contrast, the free-volume model by Turnbull and Cohen [12] predicts that the lowering of $T_{\mathrm{g}}$ would reduce the volume in a glassy state, which is seemingly opposite to those observations. We may then envisage different behaviors in the thermal and the electronic glass-transition. On the other hand, regarding the specific heat, to the authors' knowledge, there have been no reports of in situ calorimetric measurements which substantiate $T_{\mathrm{g}}$ lowering (e.g., to room temperature in $\mathrm{As}_{2} \mathrm{~S}_{3}$ ) during light irradiation. Probably, ultrahigh-sensitive scanning calorimeters with specially-designed sample pans are required for such experiments. Incidentally, relations between the photoinduced elasticity [52] and the fluidity also remain to be studied. In short, the electronic glass-transition is still elusive, which appears to be a tempting, while challenging concept for the future.

\section{Conclusions}

We have studied elemental processes giving rise to the photoinduced fluidity in chalcogenide glasses through analyses of photo-structural responses in Se clusters using an ab initio MO software. The calculation has demonstrated that the disordered structures as the followings have smaller optical gaps: i) bent and stretched segments having strong intra-cluster LP-LP interactions and ii) dimeric structures intensely interacting through inter-cluster LP-LP wavefunctions. In retrospect, it has been assumed that the excitation of LP electrons in LP semiconductors does not necessarily cut covalent bonds [12]. However, it seems that such disordered sites as above in real a-Se are excited by sub-gap photons, followed by bond ruptures, which can release strains with reduction of Se-chain lengths. Taking the Rouse model proposed for $\mathrm{PE}$ into account, we assume that the chain fragmentation causes of the fluidity. Besides, chain crossing through photo-production of valence alternation pairs can assist to untie entangled chain knots.

If the photoinduced fluidity represents the electronic glass-transition awaits further studies.

The authors would like to thank Dr. M. Repka and Professor T. Wagner for private communications. 


\section{References}

[1] O.U. Vonwiller, Nature (London) 104 (1919) 345.

[2] K. Zheng, C. Wang, Y.-Q. Cheng, Y. Yue, X. Han, Z. Zhang, Z. Shan, S.X. Mao, M. Ye, Y. Yin, E. Ma, Nat. Commun. 1021 (2010) 1-24.

[3] H. Koseki, A. Odajima, Jpn. J. Appl. Phys. 21 (1982) 424-428.

[4] H. Hisakuni, K. Tanaka, Science 270 (1995) 974-975.

[5] K. Tanaka, C.R. Chimie 5 (2002) 805-811.

[6] M. Repka, M. Frumar, M. Hrdlicka, J. Phys. Chem. Solids 68 (2007) 940-942.

[7] Y. Gueguen, J.C. Sangleboeuf, V. Keryvin, E. Lépine, Z. Yang, T. Rouxel, C. Point, B. Bureau, X.-H. Zhang, P. Lucas, Phys. Rev. B 82 (2010) 134114.

[8] Y. Gueguen, E.A. King, V. Keryvin, J.C. Sangleboeuf, T. Rouxel, B. Bureau, P. Lucas, J. Appl. Phys. 114 (2013) 074901.

[9] J.A. Van Vechten, R. Tsu, F.W. Saris, Phys. Lett. 74A (1979) 422-426.

[10] T. Gorkhover, S. Schorb, R. Coffee, M. Adolph, L. Foucar, D. Rupp, A. Aquila, J.D. Bozek, S.W. Epp, B. Erk, Nat. Photonics, 10 (2016) 93-97.

[11] J. Bang, Y.Y. Sun, X.-Q. Liu, F. Gao, S.B. Zhang, Phys. Rev. Lett. 117 (2016) 126402.

[12] K. Tanaka, K. Shimakawa, Amorphous Chalcogenide Semiconductors and Related Materials, Springer, New York, 2011.

[13] E. Lepine, Z. Yang, Y. Gueguen, J. Troles, X.-H. Zhang, B. Bureau, C. Boussard-Pledel, J.-C. Sangleboeuf, P. Lucas, J. Opt. Soc. Am. B 27 (2010) 966-971.

[14] S.N. Yannoupoulos, M.L. Trunov, Phys. Stat. Sol. B 246 (2009) 1773-1785.

[15] K. Tanaka, Amorphous Chalcogenides, in: R. Wang (Ed.), Pan Stanford Pub., Singapore, 2014, (Chap. $3)$.

[16] U. Gartner, M. Reinfelde, J. Tetris, J. Optoelectron. Adv. Mater. 18 (2016) 24-28.

[17] A. H. Goldan, , C. Li, S. J. Pennycook, J. Schneider, A. Blom, W. Zhao, J. Appl. Phys. 120 (2016) 135101.

[18] Selenium, in: R.A. Zingaro, W.C. Cooper (Eds.), Van Nostrand Reinhold Company, New York, 1974.

[19] R. Brüning, E. Irving, G. LeBlanc, J. Appl. Phys. 89 (2001) 3215-3222.

[20] A.M. Saitta, M.L. Klein, J. Phys. Chem. B 104 (2000) 2197-2200.

[21] W. Paul, G.D. Smith, Rep. Prog. Phys. 67 (2004) 1117-1185.

[22] C. Baig, V.G. Mavrantzas, M. Kröger, Macromolecules 43 (2010) 6886-6902.

[23] K. M. Salerno, A. Agrawal, B.L. Peters, D. Perahia, G.S. Grest, Euero. Phys. J. Special Topics 225 (2016) 1707-1722.

[24] M.H. Nafar Sefiddshti, B.J. Edwards, B. Khomami, J. Rheol. 60 (2016) 1227-1244.

[25] R. Stadler, D.R. Bowler, D. Alfè, and M.J. Gillan, J. Phys.: Condens. Matter 12 (2000) 5109-5119.

[26] M.E. Cates, Europhys. Lett. 4 (1987) 497-502.

[27] G.J. Fang, J.E. Maclennan, Y. Yi, M.A. Glaser, M. Farrow, E. Korblova, D.M. Walba, T.E. Furtak, N.A. Clark, Nat. Commun. 4 (2013) 1521.

[28] T. Seki, Polymer J. 46 (2014) 751-768. 
[29] K. Hoshino, F. Shimojo, T. Nishida, J. Phys. Soc. Jpn. 68 (1999)1907-1911.

[30] X. Zhang, D.A. Drabold, Phys. Rev. Lett. 83 (1999) 5042-5045.

[31] J. Hegedüs, K. Kohary, D.G. Pettifor, K. Shimakawa, S. Kugler, Phys. Rev. Lett. 95 (2005) 206803.

[32] K. Prasai, P. Biswas, D.A. Drabold, Semicond. Sci. Technol. 31 (2016) 073002.

[33] M.I. Ojovan, W.E. Lee, J. Appl. Phys. 95 (2004) 3803-3810.

[34] S.V. Nemilov, J. Non-Cryst. Solids 352 (2006) 2715-2725.

[35] J.C. Mauro, R.J. Loucks, Phys. Rev. B 76 (2007) 174202.

[36] H.H. Ruan, L.C. Zhang, J. Am. Ceram. Soc. 94 (2011) 3350-3358.

[37] K. Tanaka, J. Optoelectron. Adv. Mater. 17 (2015) 1716-1727.

[38] K. Tanaka, J. Optoelectron. Adv. Mater. 19 (2017) 586-594.

[39] M. W. Schmidt, K. K. Baldridge, J. A. Boatz, S. T. Elbert, M. S. Gordon, J. H. Jensen, S. Koseki, N. Matsunaga, K. A. Nguyen, S. J. Su, T. L. Windus, M. Dupuis, J. A. Montgomery, J. Comput. Chem. 14, (1993)1347-1363.

[40] http://winmostar.com/jp/

[41] K. Tanaka, J. Non-Cryst. Solids 90 (1987) 363-370.

[42] F. Mollot, J. Cernogora, C. Benoit à la Guillaume, Philos. Mag. B 42 (1980) 643-670.

[43] A.V. Kolobov, H. Oyanagi, Ka. Tanaka, Ke. Tanaka, Phys. Rev. B 55 (1997) 26-734.

[44] A.V. Kolobov, H. Oyanagi, K. Tanaka, Phys. Rev. Lett. 87 (2001) 145502.

[45] T. Aoki, S. Komedoori, S. Kobayashi, T. Shimizu, A. Ganjoo, K. Shimakawa, J. Non-Cryst. Solids $326 \& 327$ (2003) 273-278.

[46] T. Uchino, S.R. Elliott, Phys. Rev. B 67 (2003) 174201.

[47] P. Koštál, J. Málek, J. Non-Cryst. Solids 356 (2010) 2803-2806.

[48] K. Tanaka, Solid St. Commun. 54 (1985) 867-869.

[49] L. Tichý, H. Tichá, J. Non-Cryst. Solids 189 (1995) 141-146.

[50] Y. Ikeda, K. Shimakawa, J. Non-Cryst. Solids 338-340 (2004) 539-542.

[51] M. Popescu, F. Sava, K. Shimakawa, Y. Ikeda, V. Babin, J. Optoelectron. Adv. Mater. 9 (2007) 3558-3562.

[52] J. Gump, I. Finker, H. Xia, R. Sooryakumar, W.J. Bresser, P. Boolchand, Phys. Rev. Lett. 92 (2004) 245501. 